\title{
The Application of Ant-Grey Prediction Model in Sprint Performance Prediction and the Computer Simulation
}

\author{
Tian Ligang $^{1 *}$ and Zhang Shaobin ${ }^{2}$ \\ ${ }^{1,2}$ Department of Physical Education, Hebei Agricultural University \\ tianligang1978@126.coml, ${ }^{2}$ zhangshaobin282@126.com
}

\begin{abstract}
Due to the rapid development of computer technology, the combination between college sports and the computer technology becomes a trend. In the national physical standards, sprint is a very important sport. Sprint has attracted the majority of physical education teachers and college students. In order to exercise better and more scientific, predicting the results of sprint becomes a meaningful job. Grey prediction model is one of the commonly prediction model. In the traditional Grey prediction model, we face many problems, such as the boundary value, background value problem etc. In order to solve the above problem, we propose an improved Grey prediction model. This model is ant colony-Grey prediction model (Ant-Grey prediction model). The prediction model overcomes these problems. And then we apply this model to predict the results of the sprint for the college students. In addition, we use the computer simulation.
\end{abstract}

Keywords: Ant-Grey Prediction Model, Sprint Performance Prediction, Computer Simulation

\section{Introduction}

The rapid development of computer technology brings a lot of convenience and it makes a contribution to the development of sports in a certain degree. The application of the computer technology in sports training is more and more popular. Many universities have begun to introduce the computer technology to do the scientific sports training.

On Aug 8, 7, 2003, the state general administration introduced the revision of the new national physical training standards to the community. It marked that our mass sports life entered a new standard channel. The exercise standard makes the youth, especially the college student as the focus object. When the college students actively exercise their own physical quality, their physical conditions receive more and more attention of the whole society. The sprint is a very important project in national physical training standards. Due to the characteristic of convenient exercise and wide application etc., it is not only concerned by the college students and the physical education teachers, but also many scholars. Zhang Shouwen researched and analyzed the physical active ability training of sprinters $\mathrm{f}$ Beijing partial universities [1]. Gu Zhiyong studied the training process of the skills training in the college sprint sports [2]. Wang Bo studied the psychological training of the college sprint athletes [3].

Grey model is an important branch of Grey system theory, since it was pioneered by Professor Deng in 1982[4]. Grey model is characterized by small samples, uncertainty and poor information. Nowadays, Grey model has been widely applied in various fields, including business forecasting [5-8], agriculture [9], energy demand [10], tourism[11-12], portfolio selection[13-14], transportation [15], vehicle fatality risk estimation[16], and engineering [17- 
18] etc. And these cases with incomplete or insufficient data prove that the forecasting performance of GM-related forecasting tools is better than many conventional methods.

However, the modeling mechanism of the traditional Grey prediction model has some defects, such as model boundary value problem, the background value problem and the minimum parameters estimation problem. Therefore, we use the ant colony algorithm to optimize the parameters. And we construct the optimization model based on the ant colony algorithm. In addition, we propose an improved Grey prediction model (AC-Grey prediction model). We apply the method to predict the sprint performance for the college students. The computer simulation shows that the prediction achieves good results. The structure of this paper is as follows. The first part is the introduction. The second part is the $G M(1,1)$ model. In this part, we introduce the basic theory model. The third is the defects and optimization of $G M(1,1)$ model. In this part, we indicate the defects and optimization of $G M(1,1)$ model. The fourth part is model parameter optimization based on the ant colony algorithm. In this part, we propose the improved $G M(1,1)$ model. The fifth part is the computer simulation and the last part is the conclusion.

\section{2. $G M(1,1))$ Model}

We introduce the most classic Grey model $G M(1,1)$.The first 1 means that there is one variable in this model and the other one means that we use first order differential equation.

The mean ideal of the $G M(1,1)$ is as follows.

We set $X^{(0)}=\left\{x^{(0)}(1), x^{(0)}(2), \cdots, x^{(0)}(n)\right\}$ is the original sequence and get

$$
X^{(1)}=\left\{x^{(1)}(1), x^{(1)}(2), \cdots, x^{(1)}(n)\right\}
$$

Among it

$$
x^{(1)}(k)=\sum_{i=1}^{k} x^{(0)}(i)
$$

We call the a first order linear ordinary differential equations is the albino differential equation of $G M(1,1)$

$$
\frac{d x^{(1)}}{d t}+a x^{(1)}=b
$$

The differential form is

$$
x^{(0)}(k)+a z^{(1)}(k)=b
$$

Where, $a$ is called the development coefficient which represents the development state of the prediction value. $b$ Is called the Grey action quantity and it represents change contained in the data. $a, b$ Is the first order parameter bag of GM $(1,1)$ model

$$
[a, b]^{T}=\left(B^{T} B\right)^{-1} B^{T} Y_{n}
$$

Among it

$$
\begin{aligned}
B & =\left[\begin{array}{cc}
-z^{(1)}(2) & 1 \\
-z^{(1)}(3) & 1 \\
\vdots & \vdots \\
-z^{(1)}(n) & 1
\end{array}\right] \\
Y_{n} & =\left[x^{(0)}(2), x^{(0)}(3), \cdots, x^{(0)}(n)\right]^{T}
\end{aligned}
$$

Background value is 


$$
z^{(1)}(k+1)=\frac{1}{2}\left[x^{(1)}(k)+x^{(1)}(k+1)\right] \quad k=1,2, \cdots, n-1 .
$$

The discrete solution of $G M(1,1)$ is

$$
\hat{x}^{(1)}(k+1)=\left(x^{(0)}(1)-\frac{b}{a}\right) \cdot e^{-a k}+\frac{b}{a}
$$

\section{The defects and optimization of $G M(1,1)$ model}

\subsection{The Model Boundary Value Problem}

In the traditional $G M(1,1)$ model, we can get

The precondition of $G M(1,1)$ model is $\hat{x}^{(1)}(1)=x^{(0)}(1)$.

$x^{(0)}(0)$ is not to participate the construction of ${ }_{B}$ and $Y$. Therefore, $x^{(0)}(0)$ is unrelated to the solution of the model parameter. For the value of $x^{(0)}(1)$, it cannot be ignored the index revision effect of the prediction results.

We assume that the boundary value correction formula is $x^{(1)}(1)=x^{(0)}(1)+\varepsilon$. Among them, $\varepsilon$ is the boundary value correction term.

$$
\begin{gathered}
\tilde{x}^{(1)}(k)=\left[x^{(0)}(1)+\varepsilon-\frac{b}{a}\right] e^{-a(k-1)}+\frac{b}{a} \\
\tilde{x}^{(0)}(k)\left(1-e^{a}\right)\left(x^{(0)}(1)+\varepsilon-\frac{b}{a}\right) e^{-a(k-1)}
\end{gathered}
$$

Then we can get

$$
\tilde{x}^{(1)}(k)=\hat{x}^{(1)}(k)+b e^{-a(k-1)}, \quad \tilde{x}^{(0)}(k)=\hat{x}^{(0)}(k)+b\left(1-e^{a}\right) e^{-a(k-1)}
$$

In addition, considering that the predicted results of $G M(1,1)$ model is curve fitting in the least squares, the curve does not pass $\left(1, x^{(0)}(1)\right)$. Therefore, it is lack of the theoretical basis to force the system boundary value as $x^{(1)}(1)$. That is, we limit the curve fitting by $\left(1, x^{(0)}(1)\right)$. This is one of the main error sources for $G M(1,1)$ model.

\subsection{The Background Value Problem of the Model}

In $G M(1,1)$ model, the parameter $-a$ and $b$ of the $G M(1,1)$ model is related to the construction of the background value $Z^{(1)}$. In the section $[k-1, k]$, we make the integral for the formula $\frac{d x^{(1)}}{d t}+a x^{(1)}=b$. Then we can get $\int_{k-1}^{k} \frac{d x^{(1)}(t)}{d t}+a \int_{k-1}^{k} x^{(1)}(t) d t=\int_{k-1}^{k} b d t$.

And then we get

$$
x^{(1)}(k)-x^{(1)}(k-1)+a \int_{k-1}^{k} x^{(1)}(t) d t=b
$$

(8)

Compared the type (8), we can get 


$$
z^{(1)}(k)=\int_{k-1}^{k} x^{(1)}(t) d t
$$

At the same time, when $X^{(1)}$ generates difference at $k$, from the Lagrange mean value theorem, we know that

$$
\begin{gathered}
\exists \xi_{k} \in(k-1, k), x^{(1)}(k)-x^{(1)}(k-1)=\left.\frac{d x^{(1)}}{d t}\right|_{t=\xi_{k}} \\
x^{(1)}\left(\xi_{k}\right)=\alpha_{k} x^{(1)}(k-1)+\left(1-\alpha_{k}\right) x^{(1)}(k), \alpha_{k} \in(0,1) .
\end{gathered}
$$

In the $G M(1,1)$ model, the Grey derivative $x^{(0)}(k)=x^{(1)}(k)-x^{(1)}(k-1)$ is the derivative in $\xi_{k}$. And the corresponding background value is $x^{(1)}\left(\xi_{k}\right)=\alpha_{k} x^{(1)}(k-1)+\left(1-\alpha_{k}\right) x^{(1)}(k)$. That is, the general structure of the background value is as follows.

$$
z^{(1)}(k)=\alpha_{k} x^{(1)}(k-1)+\left(1-\alpha_{k}\right) x^{(1)}(k)
$$

From the above two aspects, taking simply $z^{(1)}(k)=\frac{1}{2}\left[x^{(1)}(k)+x^{(1)}(k-1)\right]$ is not reasonable. This is one of the main error sources in the traditional $G M(1,1)$ model.

\subsection{The Least Square Parameter Estimation Problems}

In $G M(1,1)$ model, calculating parameters $\hat{A}=[\hat{a}, \hat{b}]$ follows the least squares criterion. That is, the error sum of square of the prediction results is minimum.

$$
\min _{a, b} \sum_{i=1}^{n}\left[\hat{x}^{(0)}(i)-x^{(0)}(i)\right]^{2}
$$

However, in the actual error test, we tend to value the average relative error of the predicted results minimum. That is,

$$
\min _{a, b} \sum_{i=1}^{n}\left\lceil\frac{1}{n} \sum_{i=1}^{n} \frac{\left|\hat{x}^{(0)}(i)-x^{(0)}(i)\right|}{\left|x^{(0)}(i)\right|} \%\right\} \text {. }
$$

The criterion they follow is not consistent. This is also one of the main reasons which can lead to the unqualified error test.

\section{4. $G M(1,1)$ Model Optimization Strategy}

Base on the analysis of the above $G M(1,1)$ model modeling mechanism, we can optimize $G M(1,1)$ model from two aspects.

One is to optimize $G M(1,1)$ model boundary value.

The method 1 is to add the boundary value correction and the model parameter $\varepsilon$. That is, $x^{(1)}(1)=x^{(0)}(1)+\varepsilon \quad$. Then the time response of the model becomes $\tilde{x}^{(0)}(k)=\left(1-e^{a}\right)\left(x^{(0)}(1)+\varepsilon-\frac{b}{a}\right) e^{-a(k-1)}$. We use the least square method to estimate the parameter $\varepsilon$. 
The method 2 is to select $x^{(0)}(m)$ as the system boundary value. Then the time response of the model becomes $\tilde{x}^{(0)}(k)=\left(1-e^{a}\right)\left(x^{(0)}(m)-\frac{b}{a}\right) e^{-a(k-m)}$.

The other one is to optimize the background value of the $G M(1,1)$ model.

When constructing the sequence $Z^{(1)}$ of model background value, we use $z^{(1)}(k)=\alpha_{k} x^{(1)}(k-1)+\left(1-\alpha_{k}\right) x^{(1)}(k)$. Then we use the parameter optimization method to get the parameter $\alpha_{k}$. For simplicity, we select $z^{(1)}(k)=\alpha x^{(1)}(k-1)+(1-\alpha) x^{(1)}(k)$.

To sum up, the boundary value correction is unrelated to the model parameters. After optimizing the background value, we use the least square method to solve the parameters. However, it relates to the least squares estimation problem. We consider optimizing directly the parameters of $G M(1,1)$ model time response type $\tilde{x}^{(0)}(k)=\left(1-e^{a}\right)\left(x^{(0)}(1)+\varepsilon-\frac{b}{a}\right) e^{-a(k-1)}$ which is corrected. On the one hand, we overcome $G M(1,1)$ model boundary defect. On the other hand, we avoid the background value structure and the least square parameter estimation.

We can see that there are three parameters affecting the model prediction results. They are $a, b$ and $\varepsilon$. In order to estimate these parameters, we construct an objective function. Then we use the ant colony optimization algorithm (ACA) to solve the parameters.

By 3.3, we construct the objective function by using the average relative error minimum.

$$
f(a, b, \varepsilon) \min _{a, b, \varepsilon} \sum_{i=1}^{n}\left\lfloor\frac{1}{n} \sum_{i=1}^{n} \frac{\left|\hat{x}^{(0)}(i)-x^{(0)}(i)\right|}{\left|x^{(0)}(i)\right|} \%\right\}
$$

\section{4. $G M(1,1)$ Model Parameter Optimization based on the Ant Colony}

\section{Algorithm}

The ant colony algorithm is a novel simulated evolutionary group optimization algorithm which is proposed by Italy scholar M.Dorigo. This method simulates artificially the ants to search food through the exchange of pheromone and mutual cooperation among the individual ant. Lastly, they find the shortest path from the populations to food source. It solves a series of discrete combinatorial optimization problems.

Specifically, the ants will release a substance which is called pheromone in the moving process. This pheromone is the medium for the information transmission among the ant individuals. The ant selects the walking path according to the concentrations of pheromone. If the pheromone concentration of one path is higher, the possibility of the ants select the path is greater. In addition, the pheromone will gradually decrease its volatile time. Therefore, the ant swarm behavior shows a positive feedback of a kind of information. The individual ant finds the shortest path to the source of food by the above way.

We adopt the slave swarm to estimate and optimize $G M(1,1)$ model prediction parameter $a, b$ and $\varepsilon$. The specific steps are as follows.

The step 1 is to select the number of ants $s$.

The step 2 is to estimate the range of the parameters $a, b$ and $\varepsilon$ according to the questions. 


$$
x_{a \min } \leq a \leq x_{a \max }, \quad x_{\mathrm{b}_{\min }} \leq b \leq x_{\mathrm{b} \max }, \quad x_{\varepsilon \min } \leq \varepsilon \leq x_{\varepsilon \max }
$$

Among them, $x_{\min }$ is the lowest limit of the variable value. And $x_{\max }$ is the highest limit of the variable value.

In the actual operation, we can firstly establish the traditional $G M(1,1)$ model. Then we get the estimated values of the parameters $a, b$ and select the appropriate range.

The step 3 is to subdivision the range. We divide the range of each parameter into $M$ segment. It is equivalent to divide Cartesian coordinates that the coordinate axises are $a, b$ and $\varepsilon$ into a $M \times M \times M$ matrix. The coordinate of each small square is the coordinate of the location for the ants.

$$
\left(a_{i}, b_{j}, \varepsilon_{p}\right)=\left(x_{a \min }+\frac{x_{a \max }-x_{a \min }}{M}\right) i, x_{\mathrm{b} \min }+\frac{x_{\mathrm{b}_{\max }}-x_{\mathrm{b} \min }}{M} j, x_{\varepsilon \min }+\frac{x_{\varepsilon \max }-x_{\varepsilon \min }}{M} p
$$

Among them $i, j, p=1,2, \cdots, M$.

The step 4 is to distribute randomly the initial position for the ants. At the beginning, the pheromone concentration of the matrix is equal and it is a constant. That is, the pheromone concentration is $\tau_{i j p}(0)=c$.

The step 5 is to calculate the position $\left(a_{i}, b_{j}, \varepsilon_{p}\right)$ of ants and the corresponding target value objD. The target value is the average relative error function.

$$
f(a, b, \varepsilon) \min _{a, b, \varepsilon} \sum_{i=1}^{n}\left\{\frac{1}{n} \sum_{i=1}^{n} \frac{\left|\hat{x}^{(0)}(i)-x^{(0)}(i)\right|}{\left|x^{(0)}(i)\right|} \%\right\rceil
$$

In all ants, the optimal ant is what the objective value is minimum. Then the minimum is $\operatorname{minD}$.

The step 6 is to update the pheromone concentration. The more optimal the target value, the pheromone concentration is higher. The updated formula is as follows.

$$
\tau_{i j p}(t+1)=\tau_{i j p}(t)+\frac{Q}{o b j D(i)}
$$

Among them, $Q$ is a constant. $\tau_{i j p}(t+1)$ Is the pheromone concentration of the $t+1$ cycle. With the time going, the pheromone concentration will gradually volatilize. It increases the randomness and independent of the subsequent ant's selection.

The step 7 is the ant foraging rules. Each ant searches food in the sensing range (the position is $\left.\left(a_{i}, b_{j}, \varepsilon_{p}\right)\right)$. The ants select the path according to the pheromone concentration. The stronger the pheromone concentration, the probability that the ants select is higher. The probability formula is as follows.

$$
P(i, j, p)=\frac{\tau_{i j p}}{\sum_{i, j, p} \tau_{i j p}}
$$

In addition, the ants will select other direction randomly with the small probability mistakes. At the same time, the ants can remember their own routes in order to avoid the repeat line.

The step 8 is to judgment whether the ants can meet the requirements of the precision or not. That is, we see whether it reaches the maximum iterations. If it meets the requirement, it 
outputs the optimal objective function value and the optimal position. It does not reach, and then it is back to the step 5 .

We abbreviate the above ant optimization as ACOGM model.

\section{Computer Simulation}

We choose 3 college students to predict the 50 meter sprint results including 2 male and 1 female. We collect the 50 meter sprint results of these 3 students firstly. We collect the data for 12 months. The data of first 8 months are as the samples and the data of the last four months are as the prediction data. Every student tests 3 times in one month. X1 and X2 is the male and X3 is the female. The data and the mean value of the sample are showed in the table.1. The curves of the mean value are showed in the Figure 1.

Table 1. The Data and the Mean Value of the Samples

\begin{tabular}{|c|c|c|c|c|c|c|c|c|c|c|c|c|}
\hline NO & \multicolumn{3}{|c|}{ X1 } & $\begin{array}{l}\text { mean } \\
\text { value }\end{array}$ & \multicolumn{3}{|c|}{ X2 } & $\begin{array}{l}\text { mean } \\
\text { value }\end{array}$ & \multicolumn{3}{|c|}{ X3 } & $\begin{array}{c}\text { mean } \\
\text { value }\end{array}$ \\
\hline 1 & 7.13 & 7.24 & 6.89 & 7.09 & 6.64 & 6.87 & 6.85 & 6.79 & 7.03 & 7.21 & 7.29 & 7.18 \\
\hline 2 & 7.02 & 7.05 & 6.90 & 6.99 & 6.73 & 6.51 & 6.65 & 6.63 & 6.87 & 6.93 & 7.24 & 7.01 \\
\hline 3 & 7.24 & 6.87 & 7.12 & 7.08 & 6.93 & 6.54 & 7.03 & 6.83 & 6.93 & 7.08 & 6.72 & 6.91 \\
\hline 4 & 6.71 & 7.18 & 6.93 & 6.94 & 6.85 & 6.72 & 6.56 & 6.71 & 7.28 & 6.62 & 6.97 & 6.96 \\
\hline 5 & 6.94 & 7.12 & 7.28 & 7.11 & 6.57 & 6.67 & 6.63 & 6.62 & 7.04 & 6.86 & 7.19 & 7.03 \\
\hline 6 & 6.92 & 7.23 & 7.31 & 7.15 & 6.78 & 6.61 & 6.96 & 6.78 & 7.02 & 7.30 & 6.94 & 7.09 \\
\hline 7 & 7.05 & 7.13 & 6.93 & 7.04 & 7.10 & 6.82 & 6.91 & 6.94 & 6.99 & 6.91 & 7.10 & 7.10 \\
\hline 8 & 6.98 & 6.87 & 6.86 & 6.90 & 6.95 & 6.71 & 6.63 & 6.75 & 6.60 & 7.05 & 7.03 & 6.89 \\
\hline
\end{tabular}

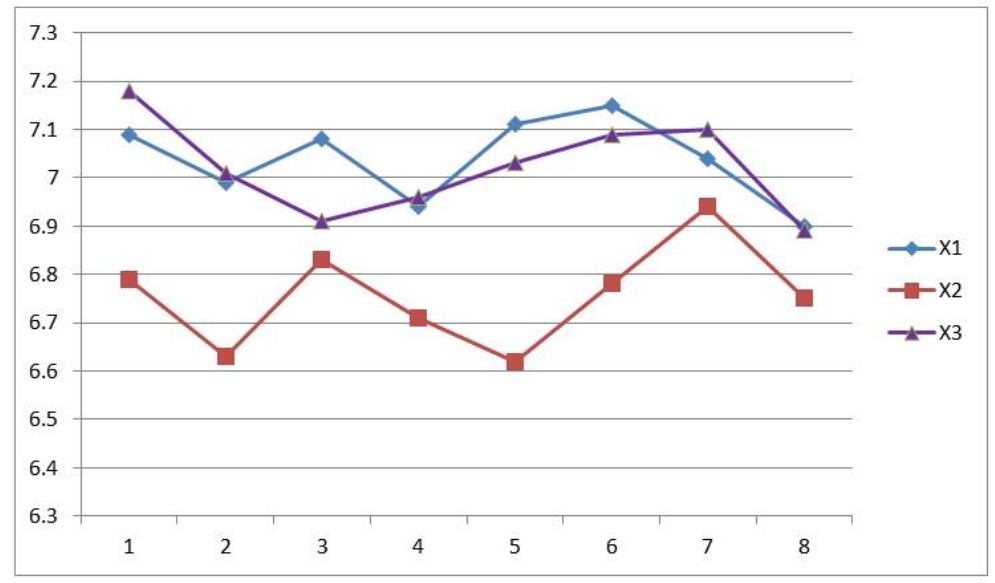

Figure 1. The Mean Value of the Sample

\subsection{Indexes Fitting}

We fit the indexes and get the fitted values. Then we calculate the error of the two columns of the values. The results are as shown in Table 2.

Table 2. The Actual Values and the Fitted Values of X1, X2 and X3

\begin{tabular}{|c|c|c|c|c|c|c|c|c|c|}
\hline \multirow{2}{*}{ NO } & \multicolumn{3}{|c|}{ X1 } & \multicolumn{3}{c|}{ X2 } & \multicolumn{3}{c|}{ X3 } \\
\cline { 2 - 8 } & AX1 & FX1 & Error & AX2 & FX2 & Error & AX3 & FX3 & Error \\
\hline
\end{tabular}




\begin{tabular}{|c|c|c|c|c|c|c|c|c|c|}
\hline & $\begin{array}{c}\text { Actual } \\
\text { values }\end{array}$ & $\begin{array}{c}\text { Fitted } \\
\text { values }\end{array}$ & $\begin{array}{c}\text { Actual } \\
\text { values }\end{array}$ & $\begin{array}{c}\text { Fitted } \\
\text { values }\end{array}$ & & $\begin{array}{c}\text { Actual } \\
\text { values }\end{array}$ & $\begin{array}{c}\text { Fitted } \\
\text { values }\end{array}$ & \\
\hline 1 & 7.09 & 7.12 & 0.0042 & 6.79 & 6.84 & 0.0073 & 7.18 & 7.21 & 0.0042 \\
\hline 2 & 6.99 & 6.87 & -0.0171 & 6.63 & 6.69 & 0.0091 & 7.01 & 6.97 & -0.0057 \\
\hline 3 & 7.08 & 7.06 & -0.0028 & 6.83 & 6.85 & 0.0030 & 6.91 & 6.94 & 0.0043 \\
\hline 4 & 6.94 & 6.98 & -0.0058 & 6.71 & 6.78 & 0.0104 & 6.96 & 7.02 & 0.0086 \\
\hline 5 & 7.11 & 7.09 & -0.0028 & 6.62 & 6.64. & 0.0030 & 7.03 & 7.01 & -0.0028 \\
\hline 6 & 7.15 & 7.16 & 0.0014 & 6.78 & 6.81 & 0.0042 & 7.09 & 7.11 & 0.0028 \\
\hline 7 & 7.04 & 7.06 & -0.0029 & 6.94 & 6.99 & 0.0072 & 7.10 & 7.13 & 0.0042 \\
\hline 8 & 6.90 & 6.93 & -0.0043 & 6.75 & 6.83 & 0.0118 & 6.89 & 6.88 & -0.0015 \\
\hline
\end{tabular}

We compare the actual values and the fitted values. The two values are drawn in the below one pictures. The results are showed in Figures 2 to 4.

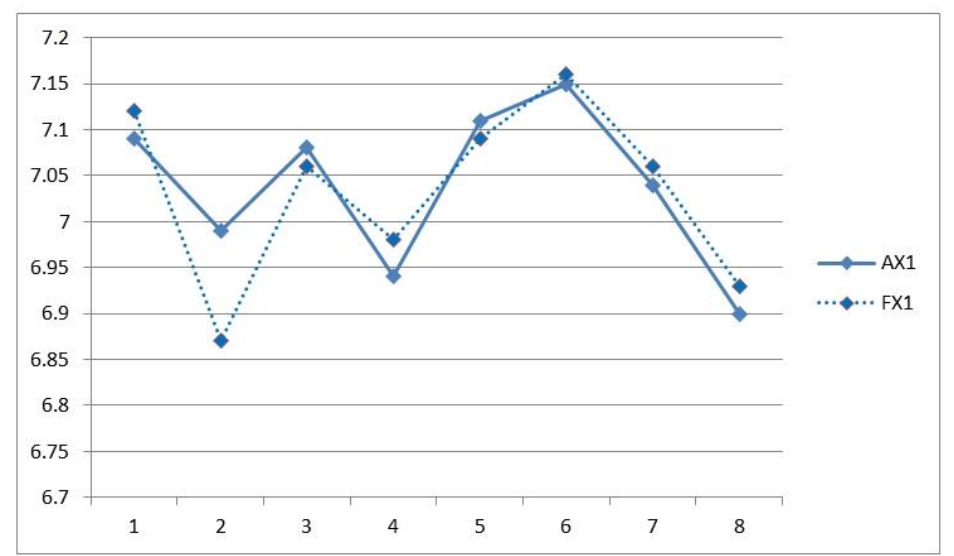

Figure 2.The Actual Values and the Fitted Values of $\mathrm{X}_{1}$

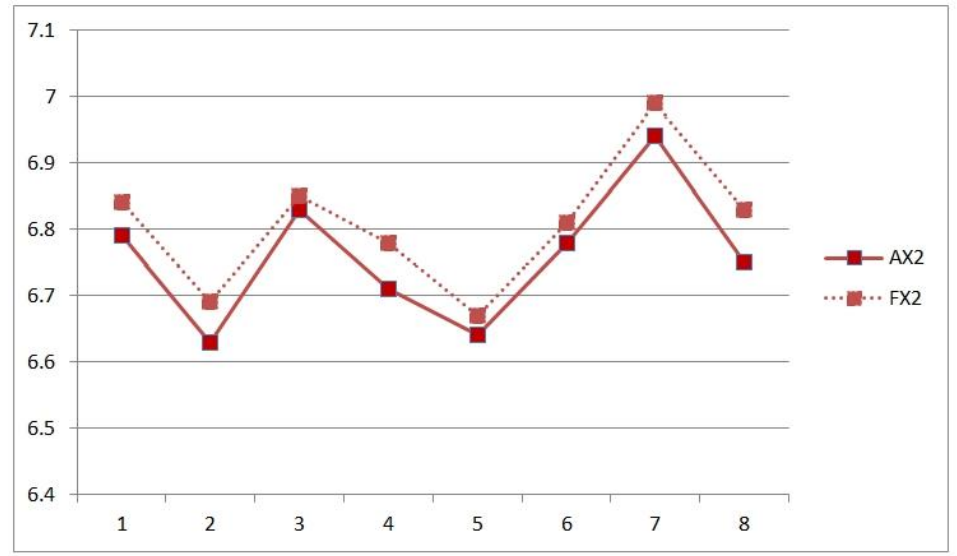

Figure 3. The Actual Values and the Fitted Values of X2 


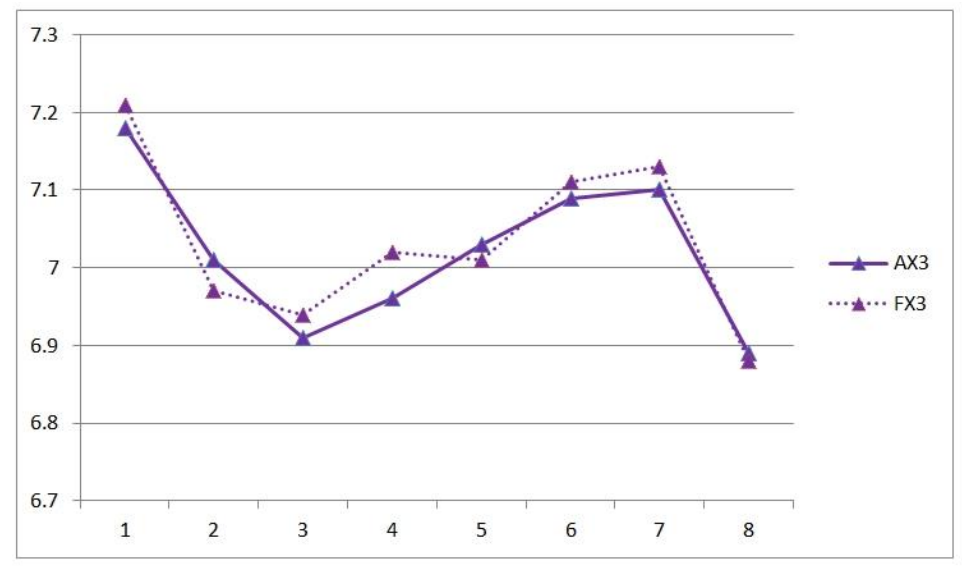

Figure 4. The Actual Values and the Fitted Values of X3

\subsection{Performance Prediction}

As the good result of fitting curves, we predict the values for the performance of the last 4 months. Then, we calculate the error between the actual values and the predicted values. AX is the actual values and the PX is the predicted values. The results are shown as the Table 3.

Table 3. The Predicted Values and the Error

\begin{tabular}{|c|c|c|c|c|c|c|c|c|c|}
\hline \multirow{3}{*}{ NO } & \multicolumn{3}{|c|}{$\mathrm{X} 1$} & \multicolumn{3}{|c|}{$\mathrm{X} 2$} & \multicolumn{3}{|c|}{$\mathrm{X} 3$} \\
\hline & AX1 & PX1 & \multirow[b]{2}{*}{ Error } & AX2 & PX2 & \multirow[b]{2}{*}{ Error } & AX3 & PX3 & \multirow[b]{2}{*}{ Error } \\
\hline & $\begin{array}{l}\text { Actual } \\
\text { values }\end{array}$ & $\begin{array}{l}\text { Predicted } \\
\text { values }\end{array}$ & & $\begin{array}{l}\text { Actual } \\
\text { values }\end{array}$ & $\begin{array}{c}\text { Predicted } \\
\text { values }\end{array}$ & & $\begin{array}{l}\text { Actual } \\
\text { values }\end{array}$ & $\begin{array}{c}\text { Predicted } \\
\text { values }\end{array}$ & \\
\hline 9 & 6.97 & 6.99 & 0.0029 & 6.74 & 6.71 & -0.0045 & 6.93 & 6.94 & 0.0014 \\
\hline 10 & 7.13 & 7.10 & -0.0042 & 6.84 & 6.83 & 0.0015 & 7.12 & 7.10 & 0.0028 \\
\hline 11 & 7.02 & 7.04 & 0.0029 & 6.71 & 6.69 & -0.003 & 7.05 & 7.05 & 0 \\
\hline 12 & 7.08 & 7.06 & -0.0028 & 6.92 & 6.94 & 0.0029 & 7.07 & 7.08 & 0.0014 \\
\hline
\end{tabular}

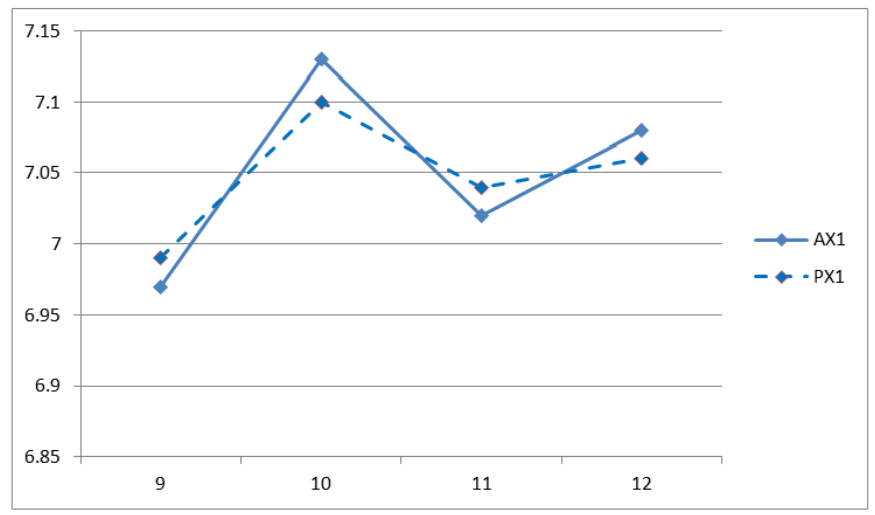

Figure 5. The Actual Values and the Predicted Values of X1 


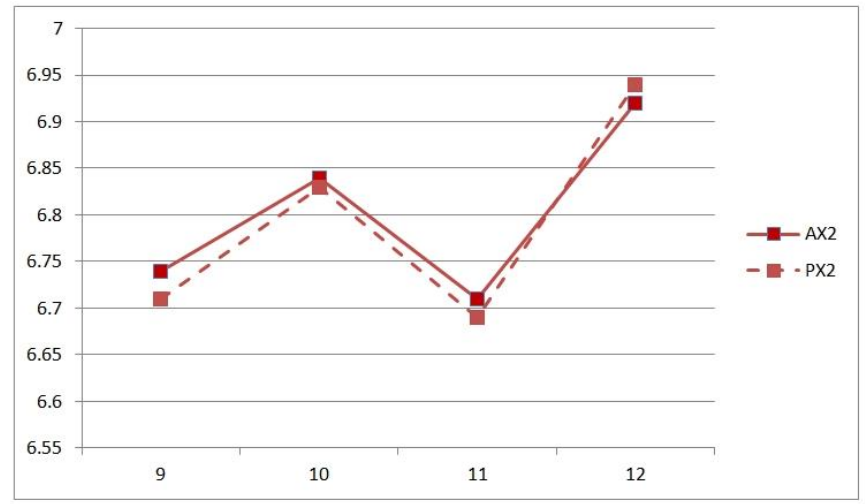

Figure 6. The Actual Values and the Predicted Values of X1

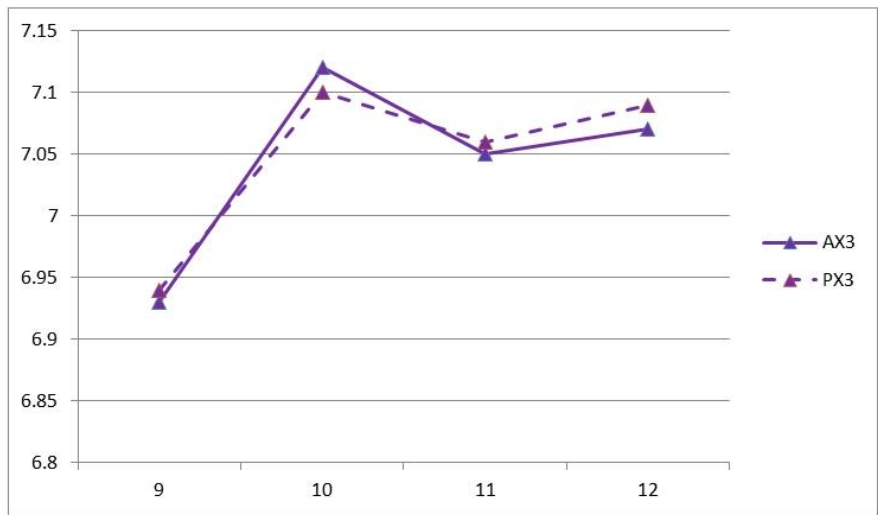

Figure 7. The Actual Values and the Predicted Values of X1

From the Figure 5 to 7 , we can see that the curve of the actual values and exactly similar with the curve of the predicted values. This means that the fitting has obtained the good effect. The AC-Grey prediction model applying to the sprint performance prediction is feasible and effective.

\section{Conclusion}

The application of computer technology in the university sports is more and more universal. The scientific exercise method and training system can improve the physical fitness and the sports performance for the college students. So, we propose an improved Grey prediction model ant colony-Grey prediction model to predict the results of the sprint. In this paper, we do some work as follows. (1)Firstly, we discuss the defects of the Grey prediction model. (2)Secondly, we propose an improved AC-Grey prediction model to optimize the parameters. (3)Thirdly, we make the computer simulation. And then we apply this improved model to predict the results of sprint for the college students. The simulation results show that the method is feasible and effective.

\section{References}

[1] S. Zhang, "The situation analysis and countermeasure research on the physical active ability training of sprinters of Beijing partial universities [C]”, Beijing Sport University, (2008). 
[2] Z. Gu, "Practice Study on Goal-setting Skills Training in Colleges and Universities Sprint Training Process [J]", Journal of Harbin Institute of Physical Education, vol. 28, no. 4, (2012), pp. 122-125.

[3] B. Wang, "Psychological training of sprint athletes in Colleges and Universities [J]", Sports World Scholarly, vol. 11, (2007), pp. 38-39.

[4] J. L. Deng, "Control problem of Grey system", Syst. Control Lett., vol. 1, (1982), pp. 288-294.

[5] Y. Zhang, J. Yang and H. Jiang, "Machine tool thermal error modeling and prediction by Grey neural network", Int. J. Adv. Manuf. Technol., vol. 59, nos. 9-12, (2012), pp. 1065-1072.

[6] C. F. Chien and K. Y. Lin, "Manufacturing intelligence for Hsinchu Science Park semiconductor sales prediction”, J. Chin. Inst. Ind. Eng., vol. 29, no. 2, (2012), pp. 98-110.

[7] Z. X. Wang, "An optimized Nash nonlinear Grey Bernoulli model for forecast-ing the main economic indices of high technology enterprises in China", Comput. Ind. Eng., vol. 64, no. 3, (2013), pp. 780-787.

[8] A. Samvedi and V. Jain, "A Grey approach for forecasting in a supply chain during intermittent disruptions", Eng. Appl. Artif. Intell., vol. 26, no. 3, (2013), pp. 1044-1051.

[9] S. L. Ou, "Forecasting agricultural output with an improved Grey forecasting model based on the genetic algorithm", Comput. Electron. Agric., vol. 85, (2012), pp. 33-39.

[10] Y. S. Lee, L. I. Tong, "Forecasting energy consumption using a Grey model improved by incorporating genetic programming", Energy Convers. Manage., vol. 52, no. 1, (2011), pp. 147-152.

[11] Y. L. Huang and Y. H. Lee, "Accurately forecasting model for the Stochastic Volatility data in tourism demand", Mod. Econ., vol. 2, no. 5, (2011), pp. 823-829.

[12] Y. L. Huang, "Forecasting the demand for health tourism in Asian countries using a GM $(1,1)$-alpha model", Tour. Hosp. Manage., vol. 18, no. 2, (2012), pp. 171-181.

[13] K. Y. Huang, C. J. Jane and T. C. Chang, "A hybrid model for portfolio selection based on Grey Relational Analysis and RS theories", In: Proceedings of the IEEE International Computer Symposium (ICS), (2010), pp. 1014-1019.

[14] C. J. Jane, "Mechanical selection system of stock portfolio based on Grey relational analysis attribute reduction mechanism”, Appl. Mech. Mater., vol. 311, (2013), pp. 73-77.

[15] Y. H. Wen, "Shipment forecasting for supply chain collaborative transportation management using Grey models with Grey numbers", Transp. Plan. Technol., vol. 34, no. 6, (2011), pp. 605-624.

[16] X. Zhu, "Application of composite Grey BP neural network forecasting model to motor vehicle fatality risk", In: Proceedings of the Second IEEE International Conference on Computer Modeling and Simulation, ICCMS'10, vol. 2, (2010), pp. 236-240.

[17] Y. Zhang, J. Yang and H. Jiang, "Machine tool thermal error modeling and prediction by Grey neural network", Int. J. Adv. Manuf. Technol., vol. 59, nos. 9-12, (2012), pp.1065-1072.

[18] T. Xia, X. Jin, L. Xi, Y. Zhang and J. Ni, "Operating load based real-time rolling Grey forecasting for machine health prognosis in dynamic maintenance schedule", (2013), J. Intell. Manuf. http://dx.doi.org/10.1007/s10845-013-0780-8.

\section{Authors}

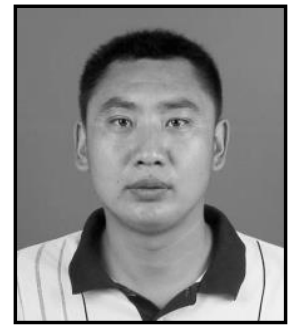

Tian Ligang, he was born in April 1978, graduated from the Hebei Institute of Physical Education for a Bachelor's Degree in 2004. He graduated from Hebei Normal University for a Master's Degree in Physical Education and Training in 2011. He is a CPC Party Member. Since 2004, Tian has been working in Department of Physical Education, Hebei Agricultural University.

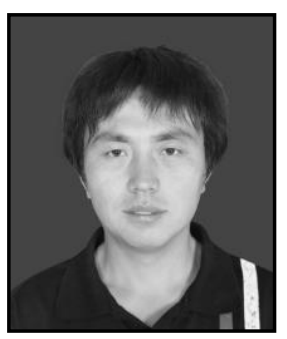

Zhang Shaobin, he was born in February 1983. He graduated from Chengdu Institute of Physical Education in July 2006. From July 2007 till now, he has been teaching physical education at universities. 
International Journal of Hybrid Information Technology Vol.8, No.2 (2015) 